\title{
Distribution of Killer Cell Immunoglobulin-Like Receptor Genes in Albanians from Republic of Macedonia
}

\author{
Aleksandar Petlichkovski ${ }^{1}$, Eli Djulejic ${ }^{2}$, Dejan Trajkov ${ }^{1}$, Olivija Efinska-Mladenovska ${ }^{1}$, Slavica Hristomanova Mitkovska ${ }^{1}$ \\ Mirko Spiroski ${ }^{1 *}$ \\ ${ }^{1}$ Institute of Immunobiology and Human Genetics, Faculty of Medicine, Ss. Cyril and Methodius University of Skopje, Skopje, \\ Republic of Macedonia; ${ }^{2}$ PAREXEL International Serbia, Takovska 46A, Belgrade 11070, Serbia
}

\begin{abstract}
Citation: Petlichkovski A, Djulejic E, Trajkov D, EfinskaMladenovska O, Hristomanova Mitkovska S, Spiroski M. Distribution of Killer Cell Immunoglobulin-Like Receptor Genes in Albanians from Republic of Macedonia. SEE J Hum Genet. 2015 Feb 19; 2015:40001. http://dx.doi.org

Key words: Killer immunoglobulin-like receptor (KIR) gene polymorphism; KIR genotyping; PCR-SSP; Albanians from

Correspondence: Prof. Dr. Mirko Spiroski. Institute of Immunobiology and Human Genetics, Faculty of Medicine, Ss Cyril and Methodius University of Skopje, 1109 Skopje, Republic or Macedonia. E-mail.mspiroski@yahoo.com Received: 28-Nov-2014; Revised: 29-Dec-2014; Accepted: 30-Jan-2015; Published: 19-Feb-2015

Copyright: @ 2015 Aleksandar Petlichkovski, Eli Djulejic, Dejan Trajkov, Olivija Efinska-Mladenovska, Slavica Hristomanova Mitkovska, Mirko Spiroski. This is an openaccess article distributed under the terms of the Creative Commons Attribution License, which permits unrestricted use, distribution, and reproduction in any medium, provided the

Competing Interests: The author have declared that no competing interests exis.
\end{abstract}

\begin{abstract}
AIM: The aim of this study was to analyze Killer Ig-Like Receptor $(K I R)$ gene polymorphisms in Albanians from Republic of Macedonia.

MATERIAL NA METHODS: The studied sample consists of 104 healthy unrelated individuals, aged 20-45 years. All individuals are of Albanian nationality, residents of different geographical regions (Skopje, Gostivar, and Tetovo) in Republic of Macedonia. The population genetics analysis package, Arlequin, was used for analysis of the data.

RESULTS: All 16 KIR genes known were observed in the Albanian individuals and framework genes (KIR3DL3, KIR3DP1, KIR2DL4, and KIR3DL2) were present in all individuals. The frequencies of other KIR genes were: KIR2DP1 (0.981), KIR2DL1 (1), KIR2DL2 (0.615), KIR2DL3 (0.865), KIR2DL5 (0.414), KIR3DL1 (0.933), KIR2DS1 (0.462), KIR2DS2 (0.606), KIR2DS3 (0.327), KIR2DS4 (0.875), KIR2DS5 (0.298), and KIR3DS1 (0.442). Tested linkage disequilibrium (LD) among $K I R$ genes demonstrated that $K I R$ genes present a wide range of linkage disequilibrium.

CONCLUSION: This is the first study analyzing the polymorphism of $K I R$ genes and genotype frequencies in Albanian individuals in the world. The results can be used for anthropological comparisons.
\end{abstract}

\section{Introduction}

The Albanians are an ethnic group inhabiting the Western Balkans that encompasses Republic of Albania and the neighbouring countries. The ancestors of the modern Albanians are considered to be the Illyrians, but admixtures with other ancient populations (for example Thracians) from the Balkans might have occurred [1]. The current size of the entire Albanian population in Europe is estimated to be around 7 million, majority of which live in the Republic of Albania (2,690,000 people) [2] and Kosovo $(1,680,000$ people) [3]. Significant Albanian populations are found in other neighbouring countries (Serbia, Montenegro, Greece, Macedonia), and there is also Albanian diaspora in some European countries, such as Italy, Germany and Switzerland.

According to the last official census from 2002, in the Republic of Macedonia, Albanians are the largest minority represented with $25.17 \%$ of the total population or 509,083 inhabitants [4]

Genetic investigations in Albanian population so far have analyzed the HLA genes distribution $[5,6]$, Y chromosome polymorphisms [7-10] and prevalence of some genetic disorders [11-13].

Killer cell immunoglobulin-like receptors (KIR) are surface molecules found on subsets of lymphoid cells. Most importantly, they influence the natural killer (NK) cells activity in activating or inhibiting manner, depending on the interaction of KIR with HLA molecules present on the target host cells $[14,15]$. The KIR locus contains a family of polymorphic and 
Table 1: Comparison of the observed and estimated $K I R$ gene frequencies for Macedonian Albanians ( $\mathrm{N}=104)$ and Native Macedonians ( $\mathrm{N}=214)$.

\begin{tabular}{|c|c|c|c|c|c|c|c|c|c|c|c|c|c|c|c|c|}
\hline & \multicolumn{16}{|c|}{ Frequencies for KIR of Macedonian Albanians and native Macedonians } \\
\hline & \multicolumn{2}{|c|}{ Pseudogenes } & \multicolumn{8}{|c|}{ Inhibitory KIR } & \multicolumn{6}{|c|}{ Non inhibitory KIR } \\
\hline & $\begin{array}{c}K I R \\
2 D P 1\end{array}$ & $\begin{array}{c}K I R \\
3 D P 1\end{array}$ & $\begin{array}{c}K I R \\
2 D L 1\end{array}$ & $\begin{array}{c}K I R \\
2 D L 2 \\
\end{array}$ & $\begin{array}{c}K I R \\
2 D L 3\end{array}$ & $\begin{array}{c}K I R \\
2 D L 4\end{array}$ & $\begin{array}{c}K I R \\
2 D L 5\end{array}$ & $\begin{array}{c}K I R \\
3 D L 1\end{array}$ & $\begin{array}{c}K I R \\
3 D L 2 \\
\end{array}$ & $\begin{array}{c}K I R \\
3 D L 3 \\
\end{array}$ & $\begin{array}{c}K I R \\
2 D S 1\end{array}$ & $\begin{array}{c}K I R \\
2 D S 2 \\
\end{array}$ & $\begin{array}{c}K I R \\
2 D S 3\end{array}$ & $\begin{array}{c}K I R \\
2 D S 4\end{array}$ & $\begin{array}{c}K I R \\
2 D S 5 \\
\end{array}$ & $\begin{array}{c}K I R \\
3 D S 1\end{array}$ \\
\hline $\begin{array}{l}\text { Macedonian } \\
\text { Albanians (N) }\end{array}$ & 102 & 104 & 104 & 64 & 89 & 104 & 43 & 97 & 104 & 104 & 48 & 63 & 34 & 91 & 31 & 46 \\
\hline $\begin{array}{l}\text { Macedonian } \\
\text { Albanians (F) }\end{array}$ & 0.981 & 1 & 1 & 0.615 & 0.865 & 1 & 0.414 & 0.933 & 1 & 1 & 0.462 & 0.606 & 0.327 & 0.875 & 0.298 & 0.442 \\
\hline $\begin{array}{l}\text { Macedonian } \\
\text { Albanians (GF) }\end{array}$ & 0.862 & 1 & 1 & 0.380 & 0.633 & 1 & 0.234 & 0.741 & 1 & 1 & 0.267 & 0.372 & 0.180 & 0.646 & 0.162 & 0.253 \\
\hline Macedonians (N) & 210 & 214 & 201 & 126 & 192 & 214 & 89 & 201 & 214 & 214 & 103 & 122 & 77 & 201 & 64 & 84 \\
\hline Macedonians (F) & 0.980 & 1 & 0.940 & 0.590 & 0.897 & 1 & 0.415 & 0.940 & 1 & 1 & 0.481 & 0.570 & 0.360 & 0.940 & 0.300 & 0.392 \\
\hline Macedonians (GF) & 0.870 & 1 & 0.760 & 0.360 & 0.690 & 1 & 0.230 & 0.800 & 1 & 1 & 0.280 & 0.350 & 0.180 & 0.800 & 0.170 & 0.220 \\
\hline Pearson's $p$ & 1 & $\&$ & 0.001 & 0.650 & 0.280 & $\&$ & 0.967 & 0.821 & $\&$ & $\&$ & 0.740 & 0.545 & 0.564 & 0.050 & 0.986 & 0.397 \\
\hline$\overline{O R}$ & 1.029 & $\&$ & $\&$ & 1.118 & 0.680 & $\&$ & 0.990 & 0.896 & $\&$ & $\&$ & 0.924 & 1.159 & 0.864 & 0.453 & 0.995 & 1.227 \\
\hline Wald $95 \% \mathrm{Cl}$ & $\begin{array}{l}0.185- \\
5.713\end{array}$ & $\&$ & $\&$ & $\begin{array}{l}0.692- \\
1.806\end{array}$ & $\begin{array}{l}0.337- \\
1.373\end{array}$ & $\&$ & $\begin{array}{l}0.615- \\
1.593\end{array}$ & $\begin{array}{l}0.347- \\
2.318\end{array}$ & $\&$ & $\&$ & $\begin{array}{l}0.578- \\
1.477\end{array}$ & $\begin{array}{l}0.719- \\
1.868\end{array}$ & $\begin{array}{l}0.526- \\
1.419\end{array}$ & $\begin{array}{l}0.202- \\
1.015\end{array}$ & $\begin{array}{l}0.596- \\
1.661\end{array}$ & $\begin{array}{l}0.764- \\
1.973 \\
\end{array}$ \\
\hline
\end{tabular}

$\mathrm{N}$, number of individuals; $\mathrm{F}$, observed frequency was obtained by direct counting; GF, gene frequencies were calculated using the formula $\mathrm{GF}=1-\sqrt{ }(1-\mathrm{F}) ; \mathrm{p}, \mathrm{statistical}$ significance; \& cannot be calculated because expected $<5, \chi 2$ test; OR, Odds ratio; $\mathrm{Cl}$, confidence interval.

highly homologous members (14 genes and 2 pseudogenes), which can be activating or inhibitory. Based on the gene content, the haplotypes have been resolved into two broad sets, termed $A$ and $B$ [16]. The different $K I R$ haplotypes vary in the number and type of genes present, but the genes KIR3DL3, KIR3DP1, KIR2DL4 and KIR3DL2 are present on virtually all haplotypes and have therefore been termed framework genes [17]. Population studies performed over the last two decades have revealed extensive diversity at the KIR gene locus, which derives from both, its polygenic and multi-allelic polymorphism, whereas on the basis of gene content, haplotype $\mathrm{B}$ displays a much greater variety of subtypes [18, 19].

The aim of this study was to examine $K I R$ gene polymorphisms by determining the frequencies of $16 K I R$ genes and pseudogenes (KIR2DL1, KIR2DL2, KIR2DL3, KIR2DL4, KIR2DL5, KIR3DL1, KIR3DL2, KIR3DL3, KIR2DS1, KIR2DS2, KIR2DS3, KIR2DS4, KIR2DS5, KIR3DS1, KIR2DP1, and $K I R 3 D P 1)$ and $K I R$ genotypes in Albanians from the Republic of Macedonia.

To our knowledge, this is the first study of the diversity of $K I R$ genes in Albanian individuals, both from the Republic of Macedonia and in the world.

\section{Material and Methods}

\section{Population samples}

The study included 104 unrelated healthy Albanian individuals (Macedonian Albanians), residents of different regions of the Republic of Macedonia (Gostivar, Skopje, Tetovo). Each individual was interviewed on a one-to-one basis; his/her genealogy was recorded for the last three generations. Admixture, if any, was recorded for each individual. Individuals with only one Albanian parent were excluded from the study. After signing of written consent, genomic DNA was extracted from the peripheral blood leukocytes using standard phenol/chloroform procedure, described elsewhere [20], and stored in the anthropology project field of the Macedonian Human DNA Bank (hDNAMKD) [21] until processing.

\section{PCR amplification}

For KIR genotyping, commercially available PEL-FREEZ KIR genotyping SSP kit (Dynal Biotech, Brown Deer, WI) was used. It is a PCR-based method (using sequence-specific priming approach) designed to detect the presence or absence of $16 \mathrm{KIR}$ genes and pseudogenes defined by the International nomenclature committee of WHO [22, 23]. In brief, locus specific primer sets, dispensed in a 96 well thermal tray were used for amplification of genomic DNA. After the amplification, the PCR products are loaded and separated by electrophoresis onto a $2 \%$ agarose gel stained with ethidium bromide, after which the results are interpreted using a worksheet for the specific amplification patterns. The presence of each KIR gene was determined by the presence of a band of DNA of the expected size.

All PCRs contained an internal positive control consisting of an additional pair of primers specific for the growth hormone $(\mathrm{GH})$ gene and a negative control [24]. Individuals were determined negative for a particular $K I R$ gene when a band of expected size was absent in the presence of a band for the $\mathrm{GH}$ gene. We have used external quality control consisting of cell lines from Immunogenetics and Histocompatibility Worskshop Conferences and Centre d' Etude du Polymorphisme Humain.

\section{Statistical analysis}

The occurrence of $K I R$ genes in individuals (frequency $=F$ ) was obtained by direct counting. Gene 
Table 2: LD analysis for KIR loci for Macedonian Albanians ( $N=104)$ and Macedonians $(N=214)$.

\begin{tabular}{|c|c|c|c|c|c|c|c|c|c|c|c|c|}
\hline \multicolumn{13}{|c|}{ Macedonian Albanians $(n=104)$} \\
\hline & KIR3DL1 & KIR2DL1 & KIR2DL3 & KIR2DS4 & KIR2DL2 & KIR2DL5 & KIR3DS1 & KIR2DS1 & KIR2DS2 & KIR2DS3 & KIR2DS5 & $K I R 2 D P 1$ \\
\hline \multicolumn{13}{|l|}{ KIR3DL1 } \\
\hline$D^{a}$ & & $\&$ & 0.0006 & 0.0589 & -0.0163 & -0.0395 & -0.0375 & -0.0362 & -0.0169 & -0.0261 & -0.0376 & -0.0013 \\
\hline$D^{\prime}$ & & $\&$ & 0.0095 & 1.0000 & -0.6286 & -1.0000 & -1.0000 & -1.0000 & -0.6376 & -0.5755 & -0.7965 & -1.0000 \\
\hline$r^{2}$ & & \& & 0.0000 & 0.5052 & 0.0178 & 0.1024 & 0.0910 & 0.0842 & 0.0191 & 0.0492 & 0.1078 & 0.0014 \\
\hline$P$ & & \& & 0.0044 & 0.0000 & 0.1734 & 0.0011 & 0.0021 & 0.0031 & 0.1588 & 0.0237 & 0.0008 & 0.7013 \\
\hline \multicolumn{13}{|l|}{ KIR2DL1 } \\
\hline$D^{a}$ & 0.0156 & & $\&$ & \& & \& & $\&$ & $\&$ & \& & $\&$ & $\&$ & $\&$ & $\&$ \\
\hline$D^{\prime}$ & 0.3225 & & $\&$ & \& & $\&$ & $\&$ & $\&$ & \& & $\&$ & $\&$ & $\&$ & $\&$ \\
\hline$r^{2}$ & 0.0871 & & \& & $\&$ & $\&$ & \& & $\&$ & $\&$ & \& & \& & $\&$ & \& \\
\hline$P$ & $<0.0001$ & & \& & $\&$ & $\&$ & \& & $\&$ & $\&$ & \& & \& & $\&$ & \& \\
\hline \multicolumn{13}{|l|}{ KIR2DL3 } \\
\hline$D$ & 0.0031 & 0.0134 & & 0.0024 & -0.0325 & -0.0213 & -0.0078 & -0.0148 & -0.0242 & -0.0329 & 0.0113 & 0.0166 \\
\hline$D^{\prime}$ & 0.0569 & 0.2907 & & 0.0222 & -0.6286 & -0.2693 & -0.1034 & -0.2041 & -0.4564 & -0.3633 & 0.2811 & 1.0000 \\
\hline$r^{2}$ & 0.0018 & 0.0400 & & 0.0005 & 0.0384 & 0.0160 & 0.0021 & 0.0076 & 0.0211 & 0.0423 & 0.0052 & 0.1261 \\
\hline$P$ & 0.5318 & 0.0034 & & 0.8281 & 0.0456 & 0.1970 & 0.6403 & 0.3753 & 0.1386 & 0.0360 & 0.4612 & 0.0003 \\
\hline \multicolumn{13}{|l|}{ KIR2DS4 } \\
\hline$D$ & 0.0480 & 0.0158 & -0.0011 & & -0.0192 & -0.0445 & -0.0601 & -0.0577 & -0.0204 & -0.0553 & -0.0493 & -0.0024 \\
\hline$D^{\prime}$ & 0.9113 & 0.3258 & -0.1894 & & -0.4000 & -0.6066 & -0.8621 & -0.8571 & -0.4146 & -0.6571 & -0.5616 & -1.0000 \\
\hline$r^{2}$ & 0.7628 & 0.0968 & 0.0002 & & 0.0143 & 0.0746 & 0.1339 & 0.1224 & 0.0160 & 0.1270 & 0.1061 & 0.0028 \\
\hline$P$ & $<0.0001$ & $<0.0001$ & 0.8192 & & 0.2229 & 0.0054 & 0.0002 & 0.0004 & 0.1973 & 0.0003 & 0.0009 & 0.5894 \\
\hline \multicolumn{13}{|l|}{ KIR2DL2 } \\
\hline$D$ & -0.0110 & -0.0165 & -0.0423 & -0.0090 & & 0.0436 & 0.0259 & 0.0237 & 0.2041 & 0.0969 & 0.0089 & -0.0074 \\
\hline$D^{\prime}$ & -0.4388 & -0.7789 & -1.0000 & -0.3920 & & 0.2744 & 0.1522 & 0.1333 & 0.8762 & 0.7706 & 0.0774 & -1.0000 \\
\hline$r^{2}$ & 0.0087 & 0.0230 & 0.0800 & 0.0064 & & 0.0332 & 0.0115 & 0.0095 & 0.7373 & 0.1803 & 0.0016 & 0.0123 \\
\hline$P$ & 0.1725 & 0.0266 & $<0.0001$ & 0.2427 & & 0.0632 & 0.2746 & 0.3196 & $<0.0001$ & $<0.0001$ & 0.6842 & 0.2589 \\
\hline \multicolumn{13}{|l|}{ KIR2DL5 } \\
\hline$D$ & -0.0215 & -0.0207 & -0.0227 & -0.0234 & 0.0822 & & 0.1633 & 0.1553 & 0.0380 & 0.0956 & 0.1268 & -0.0017 \\
\hline$D^{\prime}$ & -0.6049 & -0.6887 & -0.3775 & -0.7147 & 0.4808 & & 0.7081 & 0.6977 & 0.2331 & 0.4986 & 0.7250 & -0.1475 \\
\hline$r^{2}$ & 0.0332 & 0.0361 & 0.0229 & 0.0426 & 0.1150 & & 0.4457 & 0.4003 & 0.0249 & 0.1713 & 0.3167 & 0.0006 \\
\hline$P$ & 0.0077 & 0.0054 & 0.0268 & 0.0025 & $<0.0001$ & & $<0.0001$ & $<0.0001$ & 0.1073 & $<0.0001$ & $<0.0001$ & 0.8019 \\
\hline \multicolumn{13}{|l|}{ KIR3DS1 } \\
\hline$D$ & -0.0276 & -0.0172 & -0.0157 & -0.0294 & 0.0399 & 0.1311 & & 0.2093 & 0.0205 & 0.0958 & 0.1566 & -0.0011 \\
\hline$D^{\prime}$ & -0.7467 & -0.5510 & -0.2517 & -0.8628 & 0.2473 & 0.5720 & & 0.8789 & 0.1177 & 0.5254 & 0.9422 & -0.1034 \\
\hline$r^{2}$ & 0.0558 & 0.0255 & 0.0112 & 0.0684 & 0.0276 & 0.2969 & & 0.7147 & 0.0072 & 0.1690 & 0.4753 & 0.0003 \\
\hline$P$ & 0.0005 & 0.0196 & 0.1209 & 0.0001 & 0.0151 & $<0.0001$ & & $<0.0001$ & 0.3885 & $<0.0001$ & $<0.0001$ & 0.8683 \\
\hline \multicolumn{13}{|l|}{ KIR2DS1 } \\
\hline$D$ & -0.0315 & -0.0173 & -0.0113 & -0.0291 & 0.0390 & 0.1223 & 0.1522 & & 0.0185 & 0.0703 & 0.1605 & -0.0007 \\
\hline$D^{\prime}$ & -1.0000 & -0.6495 & -0.2113 & -1.0000 & 0.1973 & 0.5668 & 0.7475 & & 0.1016 & 0.3992 & 1.0000 & -0.0714 \\
\hline$r^{2}$ & 0.0697 & 0.0246 & 0.0055 & 0.0640 & 0.0252 & 0.2465 & 0.3891 & & 0.0058 & 0.0903 & 0.4954 & 0.0001 \\
\hline$P$ & 0.0001 & 0.0217 & 0.2774 & 0.0002 & 0.0202 & $<0.0001$ & $<0.0001$ & & 0.4389 & 0.0022 & $<0.0001$ & 0.9123 \\
\hline KIR2DS2 & & & & & & & & & & & & \\
\hline$D$ & -0.0020 & -0.0081 & -0.0447 & 0.0316 & 0.2278 & 0.0304 & -0.0077 & 0.0198 & & 0.0904 & 0.0021 & -0.0076 \\
\hline$D^{\prime}$ & -1.0000 & -1.0000 & -1.0000 & 0.1511 & 0.9799 & 0.1782 & -0.2920 & 0.1504 & & 0.7016 & 0.0181 & -1.0000 \\
\hline$r^{2}$ & 0.0036 & 0.0146 & 0.0881 & 0.0163 & 0.8725 & 0.0158 & 0.0042 & 0.0076 & & 0.1556 & 0.0001 & 0.0128 \\
\hline$P$ & 0.3795 & 0.0767 & $<0.0001$ & 0.0620 & $<0.0001$ & 0.0662 & 0.3409 & 0.2027 & & 0.0001 & 0.9227 & 0.2493 \\
\hline KIR2DS3 & & & & & & & & & & & & \\
\hline$D$ & -0.0109 & 0.0091 & -0.0424 & -0.0079 & 0.1153 & 0.1074 & 0.0690 & 0.0605 & 0.0779 & & -0.0013 & 0.0063 \\
\hline$D^{\prime}$ & -0.2791 & 0.4947 & -0.6450 & -0.2190 & 0.7789 & 0.5109 & 0.3159 & 0.3240 & 0.4312 & & -0.0133 & 1.0000 \\
\hline$r^{2}$ & 0.0090 & 0.0075 & 0.0848 & 0.0051 & 0.2382 & 0.2060 & 0.0868 & 0.0636 & 0.1017 & & 0.0000 & 0.0095 \\
\hline$P$ & 0.1661 & 0.2066 & $<0.0001$ & 0.2977 & $<0.0001$ & $<0.0001$ & $<0.0001$ & 0.0002 & $<0.0001$ & & 0.9509 & 0.3196 \\
\hline KIR2DS5 & & & & & & & & & & & & \\
\hline$D$ & -0.0330 & -0.0171 & -0.0108 & -0.0297 & 0.0314 & 0.1027 & 0.1284 & 0.1482 & 0.1190 & -0.0065 & & -0.0039 \\
\hline$D^{\prime}$ & -0.7790 & -0.4777 & -0.1513 & -0.7606 & 0.2517 & 0.5786 & 0.6961 & 0.9407 & 0.7609 & -0.0593 & & -0.2877 \\
\hline$r^{2}$ & 0.0900 & 0.0283 & 0.0060 & 0.0788 & 0.0193 & 0.2051 & 0.3271 & 0.4160 & 0.2501 & 0.0009 & & 0.0038 \\
\hline$P$ & $<0.0001$ & 0.0138 & 0.2566 & $<0.0001$ & 0.0421 & $<0.0001$ & $<0.0001$ & $<0.0001$ & $<0.0001$ & 0.6673 & & 0.5284 \\
\hline$K I R 2 D P 1$ & & & & & & & & & & & & \\
\hline$D$ & -0.0011 & 0.0131 & 0.0168 & -0.0010 & -0.0077 & -0.0016 & -0.0067 & -0.0003 & -0.0447 & 0.0067 & -0.0037 & \\
\hline$D^{\prime}$ & -1.0000 & 0.7365 & 1.0000 & -1.0000 & -1.0000 & -0.1440 & -0.5885 & -0.0360 & -1.0000 & 1.0000 & -0.2819 & \\
\hline$r^{2}$ & 0.0012 & 0.1906 & 0.1662 & 0.0011 & 0.0133 & 0.0006 & 0.0102 & 0.0000 & 0.0881 & 0.0107 & 0.0035 & \\
\hline$P$ & 0.6076 & $<0.0001$ & $<0.0001$ & 0.6227 & 0.0916 & 0.7304 & 0.1394 & 0.9398 & $<0.0001$ & 0.1301 & 0.3889 & \\
\hline KIR3DP1 & & & & & & & & & & & & \\
\hline$D$ & -0.0003 & -0.0002 & 0.0042 & -0.0003 & -0.0019 & 0.0019 & -0.0028 & 0.0022 & -0.0177 & 0.0017 & 0.0014 & 0.0046 \\
\hline$D^{\prime}$ & -1.0000 & -1.0000 & 1.0000 & -1.0000 & -1.0000 & 1.0000 & -1.0000 & 1.0000 & -0.7908 & 1.0000 & 1.0000 & 1.0000 \\
\hline$r^{2}$ & 0.0003 & 0.0003 & 0.0410 & 0.0003 & 0.0033 & 0.0033 & 0.0073 & 0.0044 & 0.0260 & 0.0026 & 0.0020 & 0.2465 \\
\hline$P$ & 0.7988 & 0.8155 & 0.0031 & 0.8070 & 0.4022 & 0.3977 & 0.2124 & 0.3344 & 0.0182 & 0.4524 & 0.5079 & $<0.0001$ \\
\hline & & & & & & onians ( $\mathrm{n}$ & & & & & & \\
\hline
\end{tabular}

$\left(D_{\text {max }}\right)$, given the allele frequencies; $r^{2}$, another way to standardise the simple measure of linkage disequilibrium [29]; $P$, statistical significance. \&, not calculated for Macedonian Albanians.

frequencies (GF) were calculated using the formula $G F=1-\sqrt{ }(1-F)$, being aware of the limitation in its ability to detect KIR genes present at low frequency. For analysis of the molecular polymorphism of the locus studied, the Arlequin software version 3.0 [25] was used.

Linkage disequilibrium (LD) values for two locus associations were calculated using $2 \times 2$ tables [26]. Because LD is not independent of allele frequencies, normalized LD was calculated as described previously [27, 28]. Comparisons of different genotypes for two groups were tested by the $\chi 2$ test. Crude odds ratios (OR) were calculated within $95 \% \mathrm{Cl}$. Previously published results for KIR gene polymorphisms in Macedonian population, [29] were used for comparison with KIR gene frequencies of the studied Albanian population.

\section{Results}

\section{KIR gene frequencies}

The presence and absence of the $16 \mathrm{KIR}$ genes (14 genes and 2 pseudogenes) determined in the 104 healthy individuals, randomly selected from 
Table 3: Pairs of KIR loci that displayed significant $(\mathrm{p}<0.05)$ LD in Macedonian Albanians $(\mathrm{N}=104)$ and Macedonians $(\mathrm{N}=214)$.

\begin{tabular}{|c|c|c|c|c|c|c|c|c|c|c|c|c|}
\hline \multicolumn{13}{|c|}{ Macedonian Albanians $(n=104)$} \\
\hline & KIR3DL1 & KIR2DL1 & KIR2DL3 & KIR2DS4 & KIR2DL2 & KIR2DL5 & KIR3DS1 & KIR2DS1 & KIR2DS2 & KIR2DS3 & KIR2DS5 & $K I R 2 D P 1$ \\
\hline KIR3DL1 & & $\&$ & + & + & 0 & - & - & - & 0 & - & - & 0 \\
\hline KIR2DL1 & + & & \& & \& & \& & \& & \& & \& & \& & \& & \& & \& \\
\hline KIR2DL3 & 0 & + & & 0 & - & 0 & 0 & 0 & 0 & - & 0 & + \\
\hline KIR2DS4 & + & + & 0 & & 0 & - & - & - & 0 & - & - & 0 \\
\hline KIR2DL2 & 0 & - & - & 0 & & 0 & 0 & 0 & + & + & 0 & 0 \\
\hline KIR2DL5 & - & - & - & - & + & & + & + & 0 & + & + & 0 \\
\hline KIR3DS1 & - & - & 0 & - & + & + & & + & 0 & + & + & 0 \\
\hline KIR2DS1 & - & - & 0 & - & + & + & + & & 0 & + & + & 0 \\
\hline KIR2DS2 & 0 & 0 & - & 0 & + & 0 & 0 & 0 & & + & 0 & 0 \\
\hline KIR2DS3 & 0 & 0 & - & 0 & + & + & + & + & + & & 0 & 0 \\
\hline KIR2DS5 & - & - & 0 & - & + & + & + & + & + & 0 & & 0 \\
\hline KIR2DP1 & 0 & + & + & 0 & 0 & 0 & 0 & 0 & - & 0 & 0 & \\
\hline KIR3DP1 & 0 & 0 & + & 0 & 0 & 0 & 0 & 0 & - & 0 & 0 & + \\
\hline
\end{tabular}

0, no significant LD; +, significant positive LD; -, significant negative LD; \&, LD not calculated.

the Macedonian Albanians is shown in Table 1, along with the corresponding frequencies for the KIR genes in the native Macedonians [29]. All $16 \mathrm{KIR}$ genes were observed in the Macedonian Albanians and framework genes (KIR3DL3, KIR3DP1, KIR2DL4, and $K I R 3 D L 2)$ were present in all individuals. The frequencies of other KIR genes were: KIR2DP1 (0.981), KIR2DL1 (1), KIR2DL2 (0.615), KIR2DL3 (0.865), KIR2DL5 (0.414), KIR3DL1 (0.933), KIR2DS1 (0.462), KIR2DS2 (0.606), KIR2DS3 (0.327), KIR2DS4 (0.875), KIR2DS5 (0.298), and KIR3DS1 (0.442). The corresponding calculated gene frequencies were: KIR2DP1 (0.862), KIR2DL1 (1), KIR2DL2 (0.380), KIR2DL3 (0.633), KIR2DL5 (0.234), KIR3DL1 (0.741), KIR2DS1 (0.267), KIR2DS2 (0.372), KIR2DS3 (0.180), KIR2DS4 (0.646), KIR2DS5 (0.162), and KIR3DS1 (0.253).

Comparison of KIR gene frequencies between Macedonian Albanians and Macedonians reveals statistically significant differences for KIR2DL1 $(\mathrm{p}=0.001)$ and KIR2DS4 $(\mathrm{p}=0.050)($ Table 1$)$.

\section{Linkage Disequilibrium}

The classical linkage disequilibrium coefficient $(D)$, linkage disequilibrium coefficient $D$ standardized by the maximum value it can take $\left(D_{\max }\right)$, given the allele frequencies $\left(D^{\prime}\right)$, standardised simple measure of linkage disequilibrium $\left(r^{2}\right)$, and statistical significance $(P)$ for KIR genes are shown in Table 2. The genes present in all individuals (KIR3DP1, KIR2DL4, KIR3DL2 and KIR3DL3) were excluded from the analysis.

Pairs of KIR loci that displayed the most significant $(P<0.05)$ LD in Macedonian Albanians are given in Table 3 . The most striking positive LD $(P<$ 0.0001 ) was observed between pairs KIR3DL1 and KIR2DS4, KIR2DL1 and KIR3DL1, KIR2DS4 with KIR3DL1 and KIR2DL1, KIR2DL2 with KIR2DS2 and KIR2DS3. Furthermore, KIR2DL5 was in positive LD with KIR2DL2 and several activating genes (KIR3DS1, KIR2DS1, KIR2DS3, and KIR2DS5), very similar to KIR3DS1 which was in highly significant positive LD with KIR2DL5, KIR2DS1, KIR2DS3, and
KIR2DS5. This pattern of highly significant LD within several pairs of activating KIR genes was also observed for KIR2DS1, KIR2DS3 and KIR2DS5. As for negative $L D$, the highest significance $(P<0.0001)$ was observed between pairs KIR2DL2 and KIR2DL3, $K I R 2 D S 2$ and $K I R 2 D L 2, K I R 2 D S 5$ with $K I R 3 D L 1$ and $K I R 2 D S 4$, and also for KIR2DP1 and KIR2DS2.

\section{Genotype frequencies}

KIR groups, genotype ID, KIR genotypes, number of individuals displaying certain genotype, and the frequency of genotypes are given in Table 4.

If any of the genes 2DL2, 2DL5, 3DS1, $2 D S 1,2 D S 2$, 2DS3, or 2DS5 was present; the genotype was considered as $\mathrm{B}$. If none of these were present, genotype is considered as AA. We have not attempted to distinguish between $A B$ and $B B$ genotypes and called any of this $\mathrm{Bx}$. KIR genotypes were numerated according to the Allelefrequencies $K I R$ Database [30, 31]. Total of 45 different $K I R$ genotypes were found to be present in studied population, based on the presence of $16 \mathrm{KIR}$ genes. We have found two group AA genotype (genotype ID 1 and 180) with frequency of 0.192 and 0.010 respectively, and 43 group $\mathrm{Bx}$ genotypes. The most frequent genotypes in the $\mathrm{Bx}$ group were genotypes ID 4 with frequency of 0.164 , and ID 2 with frequency of 0.067 . Five new genotypes of the $B x$ group were found (Table 4).

There is not statistically significant difference in distribution of $\mathrm{AA}$ and $\mathrm{Bx}$ KIR genotypes between Macedonian Albanians and native Macedonians ( $P=$ 0.789$, OR $=0.924$, Wald $95 \% \mathrm{Cl}=0.518-1.649)$ (Table 5).

\section{Discussion}

We present for the first time $K I R$ genes distribution in Albanian individuals living in the Republic of Macedonia. Albanians have probably 
Table 4: KIR locus haplogroups, genotypes ID and genotype frequency of Macedonian Albanians $(\mathrm{N}=104)$ and Macedonians $(\mathrm{N}=$ 214).

\begin{tabular}{|c|c|c|c|c|c|c|c|c|c|c|c|c|c|c|c|c|c|c|c|c|}
\hline $\begin{array}{l}\text { Haplo } \\
\text { group }\end{array}$ & Genotype ID & $\begin{array}{c}K I R \\
3 D L 1\end{array}$ & $\begin{array}{l}K I R \\
2 D L 1\end{array}$ & $\begin{array}{c}K I R \\
2 D L 3\end{array}$ & $\begin{array}{c}K I R \\
2 D S 4\end{array}$ & $\begin{array}{l}K I R \\
2 D L 2\end{array}$ & $\begin{array}{c}K I R \\
2 D L 5\end{array}$ & $\begin{array}{l}K I R \\
3 D S 1\end{array}$ & $\begin{array}{l}K I R \\
2 D S 1\end{array}$ & $\begin{array}{c}K I R \\
2 D S 2\end{array}$ & $\begin{array}{c}K I R \\
2 D S 3\end{array}$ & $\begin{array}{c}K I R \\
2 D S 5\end{array}$ & $\begin{array}{c}K I R \\
2 D L 4\end{array}$ & $\begin{array}{c}K I R \\
3 D L 2\end{array}$ & $\begin{array}{l}K I R \\
3 D L 3 \\
\end{array}$ & $\begin{array}{c}K I R \\
2 D P 1\end{array}$ & $\begin{array}{c}K I R \\
3 D P 1 \\
\end{array}$ & Macedonian Albanians No (F) & $\begin{array}{l}\text { Macedonians } \bar{F} \\
\quad \text { No }(F)\end{array}$ & $\begin{array}{c}\text { Pearson's } \\
P\end{array}$ \\
\hline AA & 1 & 1 & 1 & $\frac{2 L \mathrm{~L}}{1}$ & 1 & 0 & 0 & $\frac{0}{0}$ & 0 & 0 & 0 & 0 & 1 & 1 & 1 & 1 & 1 & $20(0.192)$ & $46(0.215)$ & 0.640 \\
\hline AA & 180 & 1 & 1 & 0 & 1 & 0 & 0 & 0 & 0 & 0 & 0 & 0 & 1 & 1 & 1 & 1 & 1 & $1(0.010)$ & 0 & 0.098 \\
\hline $\mathrm{Bx}$ & 2 & 1 & 1 & 1 & 1 & 0 & 1 & 1 & 1 & 0 & 0 & 1 & 1 & 1 & 1 & 1 & 1 & $7(0.067)$ & $11(0.051)$ & 0.565 \\
\hline $\mathrm{Bx}$ & 3 & 1 & 1 & 1 & 1 & 1 & 1 & 1 & 1 & 1 & 0 & 1 & 1 & 1 & 1 & 1 & 1 & $4(0.039)$ & $4(0.019)$ & 0.291 \\
\hline$\frac{D_{1}}{\mathrm{~B} x}$ & $\frac{5}{4}$ & $\frac{1}{1}$ & 1 & 1 & $\frac{1}{1}$ & 1 & 0 & 0 & 0 & $\frac{1}{1}$ & 0 & 0 & $\frac{1}{1}$ & 1 & $\frac{1}{1}$ & 1 & $\frac{1}{1}$ & $17(0.164)$ & $27(0.126)$ & 0.461 \\
\hline $\mathrm{Bx}$ & 5 & 1 & 1 & 1 & 1 & 1 & 1 & 0 & 0 & 1 & 1 & 0 & 1 & 1 & 1 & 1 & 1 & $3(0.029)$ & $\frac{8(0.120)}{8(0.037)}$ & 0.4016 \\
\hline $\mathrm{Bx}$ & 6 & 1 & 1 & 1 & 1 & 1 & 1 & 1 & 1 & 1 & 1 & 1 & 1 & 1 & 1 & 1 & 1 & $2(0.019)$ & $8(0.037)$ & 0.349 \\
\hline$\frac{D x}{B x}$ & $\frac{0}{7}$ & $\frac{1}{1}$ & $\frac{1}{1}$ & $\frac{1}{1}$ & $\frac{1}{1}$ & $\frac{1}{1}$ & $\frac{1}{1}$ & $\frac{1}{1}$ & $\frac{1}{1}$ & $\frac{1}{1}$ & $\frac{1}{1}$ & $\frac{1}{0}$ & $\frac{1}{1}$ & $\frac{1}{1}$ & $\frac{1}{1}$ & $\frac{1}{1}$ & $\frac{1}{1}$ & $\frac{10.019)}{4(0.039)}$ & $\frac{0(0.051)}{6(0.028)}$ & $\begin{array}{l}0.049 \\
0.672\end{array}$ \\
\hline $\mathrm{Bx}$ & 8 & 1 & 1 & 1 & 1 & 0 & 1 & 1 & 1 & 0 & 1 & 0 & 1 & 1 & 1 & 1 & 1 & $2(0.019)$ & $2(0.009)$ & 0.458 \\
\hline $\mathrm{Bx}$ & 9 & 1 & 1 & 1 & 1 & 1 & 1 & 0 & 1 & 1 & 0 & 1 & 1 & 1 & 1 & 1 & 1 & $1(0.010)$ & $5(0.023)$ & 0.398 \\
\hline $\mathrm{Bx}$ & 10 & 1 & 1 & 1 & 1 & 0 & 0 & 0 & 0 & 1 & 0 & 0 & 1 & 1 & 1 & 1 & 1 & $2(0.019)$ & $1(0.005)$ & 0.208 \\
\hline$\frac{D x}{B x}$ & $\frac{10}{11}$ & $\frac{1}{1}$ & $\frac{1}{1}$ & $\frac{1}{1}$ & $\frac{1}{1}$ & $\frac{0}{1}$ & $\frac{0}{1}$ & 0 & 1 & $\frac{1}{1}$ & $\frac{0}{1}$ & 0 & $\frac{1}{1}$ & $\frac{1}{1}$ & $\frac{1}{1}$ & $\frac{1}{1}$ & $\frac{1}{1}$ & $\frac{2(0.0)}{0}$ & $\frac{0.005)}{6(0.028)}$ & $\begin{array}{l}. \angle 026 \\
0.026\end{array}$ \\
\hline $\mathrm{Bx}$ & 13 & 1 & 1 & 1 & 1 & 1 & 1 & 1 & 0 & 1 & 1 & 0 & 1 & 1 & 1 & 1 & 1 & $1(0.010)$ & $6(0.028)$ & 0.294 \\
\hline $\mathrm{Bx}$ & 14 & 1 & 1 & 1 & 1 & 0 & 0 & 1 & 0 & 0 & 0 & 0 & 1 & 1 & 1 & 1 & 1 & $1(0.010)$ & $3(0.014)$ & 0.741 \\
\hline $\mathrm{Bx}$ & 15 & 1 & 1 & 1 & 1 & 0 & 0 & 0 & 1 & 0 & 0 & 0 & 1 & 1 & 1 & 1 & 1 & $1(0.010)$ & $9(0.042)$ & 0.120 \\
\hline$\frac{D x}{B x}$ & $\frac{5}{16}$ & $\frac{1}{1}$ & $\frac{1}{1}$ & $\frac{1}{1}$ & $\frac{1}{1}$ & 0 & 0 & $\frac{0}{1}$ & $\frac{1}{1}$ & 0 & 0 & 0 & $\frac{1}{1}$ & $\frac{1}{1}$ & $\frac{1}{1}$ & $\frac{1}{1}$ & $\frac{1}{1}$ & 0 & $\frac{(0.042)}{1(0.005)}$ & $\begin{array}{l}0.1<0 \\
0.367\end{array}$ \\
\hline $\mathrm{Bx}$ & 18 & 1 & 1 & 1 & 1 & 1 & 1 & 1 & 1 & 0 & 0 & 1 & 1 & 1 & 1 & 1 & 1 & $1(0.010)$ & $2(0.009)$ & 1 \\
\hline $\mathrm{Bx}$ & 19 & 1 & 1 & 1 & 1 & 1 & 0 & 0 & 0 & 0 & 0 & 0 & 1 & 1 & 1 & 1 & 1 & $1(0.010)$ & $1(0.005)$ & 0.601 \\
\hline $\mathrm{Bx}$ & 23 & 1 & 1 & 1 & 1 & 0 & 0 & 0 & 0 & 0 & 0 & 1 & 1 & 1 & 1 & 1 & 1 & 0 & $1(0.005)$ & 0.367 \\
\hline $\mathrm{Bx}$ & 28 & 1 & 1 & 1 & 1 & 0 & 1 & 1 & 1 & 0 & 1 & 1 & 1 & 1 & 1 & 1 & 1 & 0 & $1(0.005)$ & 0.367 \\
\hline $\mathrm{Bx}$ & 31 & 1 & 1 & 1 & 1 & 1 & 1 & 0 & 0 & 1 & 0 & 0 & 1 & 1 & 1 & 1 & 1 & $1(0.010)$ & 0 & 0.098 \\
\hline $\mathrm{Bx}$ & 33 & 1 & 1 & 1 & 1 & 0 & 1 & 1 & 1 & 0 & 0 & 0 & 1 & 1 & 1 & 1 & 1 & 0 & $1(0.005)$ & 0.367 \\
\hline $\mathrm{Bx}$ & 41 & 1 & 1 & 1 & 1 & 1 & 0 & 1 & 0 & 1 & 1 & 0 & 1 & 1 & 1 & 1 & 1 & $1(0.010)$ & 0 & 0.098 \\
\hline $\mathrm{Bx}$ & 62 & 1 & 1 & 1 & 1 & 1 & 0 & 0 & 0 & 1 & 1 & 0 & 1 & 1 & 1 & 1 & 1 & $3(0.029)$ & $3(0.014)$ & 0.0362 \\
\hline $\mathrm{Bx}$ & 63 & 1 & 1 & 1 & 1 & 1 & 0 & 1 & 1 & 1 & 0 & 1 & 1 & 1 & 1 & 1 & 1 & $1(0.010)$ & $2(0.009)$ & 1 \\
\hline $\mathrm{Bx}$ & 68 & 0 & 1 & 1 & 0 & 1 & 1 & 1 & 1 & 1 & 0 & 1 & 1 & 1 & 1 & 1 & 1 & $1(0.010)$ & 0 & 0.098 \\
\hline $\mathrm{Bx}$ & 69 & 0 & 1 & 1 & 0 & 0 & 1 & 1 & 1 & 0 & 0 & 1 & 1 & 1 & 1 & 1 & 1 & 0 & $2(0.009)$ & 0.306 \\
\hline$\frac{D x}{B x}$ & $\frac{55}{70}$ & $\frac{0}{0}$ & $\frac{1}{1}$ & $\frac{1}{1}$ & 0 & $\frac{0}{1}$ & $\frac{1}{1}$ & $\frac{1}{1}$ & $\frac{1}{1}$ & $\frac{0}{1}$ & $\frac{0}{1}$ & $\frac{1}{1}$ & $\frac{1}{1}$ & $\frac{1}{1}$ & $\frac{1}{1}$ & $\frac{1}{1}$ & $\frac{1}{1}$ & $3(0.029)$ & $\frac{2(0.005)}{2(0.009)}$ & 0.000 \\
\hline $\mathrm{Bx}$ & 71 & 1 & 1 & 0 & 1 & 1 & 1 & 0 & 0 & 1 & 1 & 0 & 1 & 1 & 1 & 1 & 1 & $2(0.019)$ & $4(0.019)$ & 1 \\
\hline $\mathrm{Bx}$ & 72 & 1 & 0 & 0 & 1 & 1 & 0 & 0 & 0 & 1 & 0 & 0 & 1 & 1 & 1 & 0 & 1 & 0 & $1(0.005)$ & 0.367 \\
\hline $\mathrm{Bx}$ & 73 & 1 & 1 & 0 & 1 & 1 & 1 & 1 & 1 & 1 & 1 & 1 & 1 & 1 & 1 & 1 & 1 & $1(0.010)$ & $5(0.023)$ & 0.398 \\
\hline $\mathrm{Bx}$ & 76 & 1 & 0 & 0 & 1 & 1 & 1 & 1 & 1 & 1 & 0 & 1 & 1 & 1 & 1 & 0 & 1 & 0 & $2(0.009)$ & 0.306 \\
\hline $\mathrm{Bx}$ & 79 & 1 & 1 & 1 & 0 & 0 & 1 & 1 & 1 & 0 & 0 & 1 & 1 & 1 & 1 & 1 & 1 & $1(0.010)$ & 0 & 0.098 \\
\hline $\mathrm{Bx}$ & 87 & 0 & 1 & 1 & 1 & 1 & 1 & 1 & 1 & 1 & 1 & 1 & 1 & 1 & 1 & 1 & 1 & 0 & $1(0.005)$ & 0.470 \\
\hline $\mathrm{Bx}$ & 88 & 0 & 1 & 1 & 0 & 0 & 1 & 1 & 1 & 1 & 0 & 1 & 1 & 1 & 1 & 1 & 1 & $1(0.010)$ & 0 & 0.098 \\
\hline $\mathrm{Bx}$ & 90 & 1 & 1 & 0 & 1 & 1 & 1 & 1 & 1 & 1 & 1 & 0 & 1 & 1 & 1 & 1 & 1 & $2(0.019)$ & $2(0.009)$ & 0.458 \\
\hline $\mathrm{Bx}$ & 93 & 1 & 1 & 1 & 0 & 1 & 1 & 1 & 1 & 1 & 1 & 1 & 1 & 1 & 1 & 1 & 1 & $1(0.010)$ & 0 & 0.098 \\
\hline $\mathrm{Bx}$ & 113 & 1 & 1 & 0 & 1 & 1 & 1 & 0 & 1 & 1 & 1 & 0 & 1 & 1 & 1 & 1 & 1 & 0 & $1(0.005)$ & 0.0367 \\
\hline $\mathrm{Bx}$ & 118 & 1 & 1 & 0 & 1 & 1 & 1 & 1 & 1 & 1 & 0 & 1 & 1 & 1 & 1 & 1 & 1 & $1(0.010)$ & 0 & 0.098 \\
\hline $\mathrm{Dx}$ & 159 & 0 & 1 & 1 & 0 & 1 & 1 & 1 & 1 & 1 & 1 & 0 & 1 & 1 & $\frac{1}{1}$ & 1 & 1 & 0 & $1(0.005)$ & $\begin{array}{l}0.0367 \\
0.367\end{array}$ \\
\hline $\mathrm{Bx}$ & 171 & 1 & 1 & 0 & 1 & 1 & 0 & 0 & 0 & 0 & 0 & 0 & 1 & 1 & 1 & 1 & 1 & $1(0.010)$ & 0 & 0.098 \\
\hline $\mathrm{Bx}$ & 172 & 1 & 1 & 0 & 1 & 1 & 0 & 0 & 0 & 1 & 0 & 0 & 1 & 1 & 1 & 0 & 1 & $1(0.010)$ & 0 & 0.098 \\
\hline $\mathrm{Bx}$ & 190 & 0 & 1 & 0 & 0 & 1 & 1 & 1 & 1 & 1 & 1 & 0 & 1 & 1 & 1 & 1 & 1 & $1(0.010)$ & 0 & 0.098 \\
\hline $\mathrm{Bx}$ & 192 & 1 & 1 & 1 & 1 & 1 & 0 & 0 & 1 & 1 & 0 & 0 & 1 & 1 & 1 & 1 & 1 & $\frac{1}{2(0.019)}$ & 0 & 0.019 \\
\hline $\mathrm{Bx}$ & 200 & 1 & 1 & 1 & 1 & 0 & 1 & 0 & 0 & 0 & 0 & 0 & 1 & 1 & 1 & 1 & 1 & 0 & $1(0.005)$ & 0.367 \\
\hline $\mathrm{Bx}$ & 202 & 1 & 1 & 1 & 1 & 1 & 0 & 0 & 1 & 1 & 0 & 1 & 1 & 1 & 1 & 1 & 1 & $2(0.019)$ & $3(0.014)$ & 0.726 \\
\hline $\mathrm{Bx}$ & 205 & 1 & 1 & 1 & 1 & 0 & 0 & 0 & 1 & 0 & 1 & 0 & 1 & 1 & 1 & 1 & 1 & 0 & $1(0.005)$ & 0.367 \\
\hline $\mathrm{Bx}$ & 233 & 1 & 1 & 1 & 1 & 1 & 0 & 1 & 1 & 1 & 1 & 0 & 1 & 1 & 1 & 1 & 1 & $1(0.010)$ & $5(0.023)$ & 0.398 \\
\hline $\mathrm{Bx}$ & 260 & 1 & 1 & 1 & 1 & 0 & 0 & 0 & 0 & 0 & 1 & 0 & 1 & 1 & 1 & 1 & 1 & 0 & $2(0.009)$ & 0.306 \\
\hline$\frac{L \lambda}{B x}$ & 268 & 1 & 1 & 1 & 1 & 1 & 0 & 0 & 0 & 1 & 1 & 1 & 1 & 1 & 1 & 1 & 1 & 0 & $\frac{10.0050)}{1(0.005)}$ & $\begin{array}{l}0.000 \\
0.367\end{array}$ \\
\hline $\mathrm{Bx}$ & 294 & 0 & 1 & 1 & 0 & 1 & 1 & 1 & 1 & 0 & 1 & 1 & 1 & 1 & 1 & 1 & 1 & $1(0.010)$ & $1(0.005)$ & 0.601 \\
\hline $\mathrm{Dx}$ & 317 & 1 & 1 & 0 & 1 & $\frac{1}{1}$ & 0 & 0 & 0 & $\frac{0}{1}$ & $\frac{1}{1}$ & 0 & 1 & 1 & $\frac{1}{1}$ & 1 & 1 & 0 & $3(0.014)$ & 0.0617 \\
\hline $\mathrm{Bx}$ & 318 & 0 & 1 & 0 & 0 & 1 & 0 & 1 & 1 & 1 & 1 & 1 & 1 & 1 & 1 & 1 & 1 & 0 & $1(0.005)$ & 0.367 \\
\hline$\frac{D x}{B x}$ & $\frac{310}{319}$ & 1 & $\frac{1}{1}$ & 1 & 1 & $\frac{1}{1}$ & 0 & $\frac{1}{1}$ & $\frac{1}{1}$ & $\frac{1}{1}$ & $\frac{1}{1}$ & $\frac{1}{1}$ & $\frac{1}{1}$ & $\frac{1}{1}$ & $\frac{1}{1}$ & $\frac{1}{1}$ & $\frac{1}{1}$ & $\frac{0}{1(0.010)}$ & $\frac{10.0009)}{2(0.009}$ & $\frac{1.007}{1}$ \\
\hline $\mathrm{Bx}$ & 331 & 1 & 1 & 1 & 0 & 0 & 1 & 1 & 1 & 0 & 1 & 0 & 1 & 1 & 1 & 1 & 1 & $1(0.010)$ & 0 & 0.098 \\
\hline $\mathrm{Bx}$ & 336 & 1 & 1 & 1 & 1 & 1 & 0 & 1 & 0 & 0 & 0 & 0 & 1 & 1 & 1 & 1 & 1 & 0 & $1(0.005)$ & 0.367 \\
\hline $\mathrm{Bx}$ & 359 & 1 & 0 & 1 & 1 & 0 & 1 & 1 & 1 & 0 & 0 & 1 & 1 & 1 & 1 & 1 & 1 & 0 & $1(0.005)$ & 0.367 \\
\hline $\mathrm{Bx}$ & 363 & 1 & 0 & 1 & 1 & 1 & 1 & 1 & 1 & 1 & 0 & $\frac{1}{1}$ & $\frac{1}{1}$ & $\frac{1}{1}$ & $\frac{1}{1}$ & $\frac{1}{1}$ & $\frac{1}{1}$ & 0 & $\frac{(0.005)}{1(0.005)}$ & $\begin{array}{l}0.001 \\
0.367\end{array}$ \\
\hline $\mathrm{Bx}$ & 370 & 1 & 1 & 1 & 1 & 1 & 0 & 0 & 1 & 1 & 0 & 1 & 1 & 1 & 1 & 1 & 1 & 0 & $3(0.014)$ & 0.117 \\
\hline$\frac{D x}{B x}$ & 371 & $\frac{1}{1}$ & $\frac{1}{1}$ & $\frac{1}{1}$ & $\frac{1}{1}$ & $\frac{1}{1}$ & 0 & 0 & $\frac{1}{1}$ & $\frac{1}{0}$ & 0 & $\frac{1}{1}$ & $\frac{1}{1}$ & $\frac{1}{1}$ & $\frac{1}{1}$ & $\frac{1}{1}$ & $\frac{1}{1}$ & 0 & $\frac{10.014)}{1(0.005)}$ & 0.117 \\
\hline $\mathrm{Bx}$ & 372 & 1 & 1 & 1 & 1 & 0 & 0 & 1 & 1 & 0 & 1 & 0 & 1 & 1 & 1 & 1 & 1 & $2(0.032)$ & $1(0.005)$ & 0.208 \\
\hline$\frac{D x}{B x}$ & $\frac{316}{373}$ & $\frac{1}{1}$ & $\frac{1}{1}$ & $\frac{1}{1}$ & $\frac{1}{1}$ & $\frac{0}{1}$ & 0 & $\frac{1}{0}$ & $\frac{1}{1}$ & $\frac{0}{1}$ & $\frac{1}{1}$ & 0 & $\frac{1}{1}$ & $\frac{1}{1}$ & $\frac{1}{1}$ & $\frac{1}{1}$ & $\frac{1}{1}$ & $\frac{2(0.00<)}{0}$ & $\frac{10.005)}{2(0.009)}$ & $\frac{0.200}{0.306}$ \\
\hline $\mathrm{Bx}$ & 374 & 1 & 0 & 1 & 0 & 1 & 1 & 1 & 1 & 1 & 1 & 0 & 1 & 1 & 1 & 1 & 1 & 0 & $1(0.005)$ & 0.367 \\
\hline $\mathrm{Bx}$ & 375 & 0 & 1 & 1 & 0 & 0 & 0 & 0 & 1 & 0 & 0 & 1 & 1 & 1 & 1 & 1 & 1 & 0 & $1(0.005)$ & 0.367 \\
\hline $\mathrm{Bx}$ & 376 & 0 & 0 & 0 & 1 & $\frac{1}{1}$ & 0 & 0 & 1 & 1 & 1 & 0 & 1 & 1 & 1 & 1 & 1 & 0 & $1(0.005)$ & 0.367 \\
\hline $\mathrm{Bx}$ & 377 & 0 & 0 & 1 & 0 & 1 & 1 & 1 & 1 & 1 & 0 & 1 & $\frac{1}{1}$ & $\frac{1}{1}$ & $\frac{1}{1}$ & $\frac{1}{1}$ & 1 & 0 & $3(0.014)$ & 0.007 \\
\hline $\mathrm{Bx}$ & 378 & 1 & 1 & 0 & 1 & 1 & 0 & 1 & 0 & 1 & 0 & 0 & 1 & 1 & 1 & 0 & 0 & 0 & $1(0.005)$ & 0.367 \\
\hline $\mathrm{Dx}$ & 379 & $\frac{1}{1}$ & 1 & 0 & 1 & 1 & 0 & 1 & 1 & 1 & 0 & 1 & 1 & 1 & $\frac{1}{1}$ & 1 & 1 & 0 & $1(0.005)$ & $\begin{array}{l}0.007 \\
\end{array}$ \\
\hline $\mathrm{Bx}$ & 380 & 1 & 0 & 1 & 1 & 1 & 1 & 0 & 0 & 1 & 0 & 0 & 1 & 1 & 1 & 1 & 1 & 0 & $1(0.005)$ & 0.367 \\
\hline $\mathrm{Bx}$ & 429 & 1 & 1 & 0 & 1 & 1 & 1 & 1 & 1 & 1 & 0 & 1 & 1 & 1 & 1 & 0 & 1 & $1(0.010)$ & 0 & 0.098 \\
\hline $\mathrm{Bx}$ & new & 1 & 1 & 1 & 0 & 1 & 0 & 1 & 1 & 1 & 1 & 1 & 1 & 1 & 1 & 1 & 1 & $1(0.010)$ & 0 & 0.098 \\
\hline$\frac{D x}{B x}$ & new & $\frac{1}{1}$ & $\frac{1}{1}$ & $\frac{1}{0}$ & $\frac{0}{1}$ & $\frac{1}{1}$ & 0 & $\frac{1}{1}$ & $\frac{1}{1}$ & $\frac{1}{1}$ & $\frac{1}{1}$ & 0 & $\frac{1}{1}$ & $\frac{1}{1}$ & $\frac{1}{1}$ & $\frac{1}{1}$ & $\frac{1}{1}$ & $\frac{(0.010)}{1(0.010)}$ & 0 & $\begin{array}{l}.050 \\
0.098\end{array}$ \\
\hline $\mathrm{Bx}$ & new & 1 & 1 & 0 & 1 & 0 & 0 & 0 & 1 & 0 & 0 & 0 & 1 & 1 & 1 & 1 & 1 & $1(0.010)$ & 0 & 0.098 \\
\hline $\mathrm{Bx}$ & new & 1 & 1 & 1 & 0 & 1 & 0 & 1 & 1 & 1 & 1 & 0 & 1 & 1 & 1 & 1 & 1 & $1(0.010)$ & 0 & 0.098 \\
\hline $\mathrm{Bx}$ & new & 1 & 1 & 0 & 0 & 1 & 0 & 0 & 0 & 1 & 1 & 0 & 1 & 1 & 1 & 1 & 1 & $1(0.010)$ & 0 & 0.098 \\
\hline
\end{tabular}

KIR Genotype [1=Positive. 0=negative]

populated the south-western part of the Balkan in the Palaeolithic Age. During the various invasions and migrations that frequently occurred in these territories, genetic admixtures within Roman and later Byzantine empires probably took place. 
When compared with the KIR gene frequencies of the native Macedonian population, the most significant difference was found for KIR2DL1 (F $=1$ in Macedonian Albanians and $F=0.940$ in Macedonians, $P=0.001)$ and for KIR2DS4 (0.875 compared to 0.940 in Macedonian population, $\mathrm{P}=$ 0.050). As expected, the frequencies of several KIR genes are comparable or even very similar with frequencies found in some Mediterranean populations, such as France, Italy, Greece, and Belgium [32-35]. Relatively high frequency of KIR2DS4 (0.875) is common for both, Macedonian Albanians and native Macedonians and it is similar to the frequencies found in populations from Belgium, England, Greece and Japan [35-37]. These findings are in agreement with previously published reports studying the HLA polymorphisms, which indicate similarity of Albanians with other Balkan and European (especially Mediterranean) ethnic groups [6].

Table 5: AA and Bx KIR haplogroup frequencies in Macedonian Albanians $(N=104)$ and Macedonians ( $N=214)$.

\begin{tabular}{lcccccccc}
\hline \multirow{2}{*}{ Haplogroup } & \multicolumn{2}{c}{ Albanians } & \multicolumn{2}{c}{ Macedonians } & \multirow{2}{*}{$\begin{array}{c}\text { Pearson's } \\
\text { p-value }\end{array}$} & Odds ratio & \multirow{2}{*}{ Wald 95\% Cl } \\
\cline { 2 - 5 } & $\mathrm{N}$ & $\mathrm{F}$ & $\mathrm{N}$ & $\mathrm{F}$ & & \\
\hline AA & 21 & 0.202 & 46 & 0.215 & & 0.789 & \multirow{2}{*}{0.924} & \multirow{2}{*}{$0.518-1.649$} \\
\hline $\mathrm{Bx}$ & 83 & 0.798 & 168 & 0.785 & & & \\
\hline
\end{tabular}

N. number of individuals displaying AA or Bx KIR genotype; F. frequency of KIR genotype; $\mathrm{Cl}$. confidence interval.

Linkage disequilibrium (LD) analysis is used in $K I R$ population studies in order to define common co segregation patterns of multiple KIR loci and also potential allelic relationships between KIR loci. We have observed several pairs in striking positive LD relationship $(P<0.0001)$ in our study, such as pairs $K I R 3 D L 1$ and KIR2DS4, KIR2DL1 and KIR3DL1, and combinations between KIR2DL5 and several activating KIR3DS1, KIR2DS1, KIR2DS3 and KIR2DS5.

Most of the observed LD patterns have been previously reported for Macedonian population (29), and Roma population from the Republic of Macedonia [38] and also other populations [39]. However, we cannot assume an absolute correlation between the $K I R$ loci, as we only detect a certain percentage of alleles at a locus.

Despite the mentioned differences in the frequencies of two KIR genes in the studied Macedonian Albanians and native Macedonian population, we have not found statistically significant differences between the two populations when comparing the frequencies of $\mathrm{AA}$ and $\mathrm{Bx} K I R$ genotypes. Similar predominance of group $B x$ genotypes has been also observed in the North Indians, Palestinians, South Asians and AfroCaribbean's [36, 39, 40]. Five new genotypes were identified and are being referred to allelefrequencies.net [30].

We published several papers about frequencies of KIR genes: in human West Nile virus infections reported 2011 in the Republic of Macedonia [41]; in Graft versus Host Disease after related haematopoietic stem cell transplantation in patients with haematological malignancies from Republic of Macedonia [42]; in pandemic influenza A (H1N1)pdm09 infection in critically ill Macedonian patients [43]; in Macedonian patients with haematological malignancies [44]; and women with infertility problems [45]. The frequency of Macedonian Albanians in these and future studies of KIR genes should be taken into account.

There are few limitations of this study that should be addressed. First, KIR typing at allelic level might be more informative but unfortunately, at present we are not able to perform it. Second, the genetic distance analysis between studied and other populations, would certainly add value to this study. However, there are recent studies arguing the real meaning and contribution of this analysis to the population comparisons, in the light of proposed different evolution of activating and inhibitory KIR genes $[46,47]$.

In conclusion, we have determined the distribution of KIR genes in Macedonian Albanians and compared it with similar results for native Macedonian populations. It would be of interest to compare these results with results for other Albanian populations in the region and throughout the world and later address the influence of migrations and admixture of populations on inheritance of $K I R$ genes. This study can be suitable for use in other anthropological studies in order to better understand genetic distances, and especially for performing a meta-analysis of KIR gene frequencies in Albanians worldwide.

\section{Acknowledgement}

The authors thank Elena Cvetkovska (Institute of Immunobiology and Human Genetics, Faculty of Medicine, Ss. Cyril i Methodius University of Skopje, Skopje, Republic of Macedonia) for isolation of genomic DNA, and taking care of the Macedonian Human DNA Bank (http://www.hdna.mk).

\section{References}

1. Demiraj S. The Origin of Albanians. Academy of Sciences of Albania, Tirana, 2006.

2. Albanian Agency for Statistics, 2011 Albania Census. Retreived June, 2012 from: https://www.cia.gov/library/publications/the-worldfactbook/geos/al.html

3. Kosovo Agency for Statistics, 2011 Kosovo Census, Retreived June, 2012 from: http://esk.rksgov.net/rekos2011/?cid=2,40,276

4. Macedonia, S. S. O. o. t. R. O. Census 2002: Total population of the Republic of Macedonia according to declared ethnic 
affiliation, Skopje, 2004.

5. Grubić Z, Kerhin-Brkljacić V, Cecuk-Jelicić E, Kuci S, Kastelan A. HLA class I polymorphism in the Albanian population. Coll Antropol. 2000;24(2):303-7.

6. Sulcebe G, Sanchez-Mazas A, Tiercy JM, Shyti E, Mone I, YIli $Z$, Kardhashi V. HLA allele and haplotype frequencies in the Albanian population and their relationship with the other European populations. Int J Immunogenet. 2009;36(6):337-43.

7. Boattini A, Luiselli D, Sazzini M, Useli A, Tagarelli G, Pettener D. Linking Italy and the Balkans. A Y-chromosome perspective from the Arbereshe of Calabria. Ann Hum Biol. 2011;38(1):5968.

8. Plaseski T, Noveski P, Popeska Z, Efremov GD, PlaseskaKaranfilska D. Association Study of Single-Nucleotide Polymorphisms in FASLG, JMJDIA, LOC203413, TEX15, BRDT, OR2W3, INSR, and TAS2R38 Genes With Male Infertility. J Androl. 2012;33(4):675-83.

9. Ferri G, Tofanelli S, Alù M, Taglioli L, Radheshi E, Corradini B, Paoli G, Capelli C, Beduschi G. Y-STR variation in Albanian populations: implications on the match probabilities and the genetic legacy of the minority claiming an Egyptian descent. Int J Legal Med. 2010;124(5):363-70.

10. Belledi M, Poloni ES, Casalotti R, Conterio F, Mikerezi I, Tagliavini J, Excoffier L. Maternal and paternal lineages in Albania and the genetic structure of Indo-European populations. Eur J Hum Genet. 2000;8(7):480-6.

11. Kruja J, Beghi E, Zerbi D, Dobi D, Kuqo A, Zekja I, Mijo S Kapisyzi M, Messina P. High prevalence of major neurological disorders in two Albanian communities: results of a door-todoor survey. Neuroepidemiology. 2012;38(3):138-47.

12. Festini F, Taccetti G, Repetto T, Mannini C, Neri S, Bisogni S, de Martino M. Incidence of cystic fibrosis in the Albanian population. Pediatr Pulmonol. 2008;43(11):1124-9.

13. Castaman G, Giacomelli SH, Ghiotto R, Boseggia C, Pojani K, Bulo $A$, Madeo D, Rodeghiero $F$. Spectrum of mutations in Albanian patients with haemophilia A: identification of ten novel mutations in the factor VIII gene. Haemophilia 2007;13(3):311-6.

14. Harel-Bellan A, Quillet A, Marchiol C, DeMars R, Tursz T, Fradelizi $D$. Natural killer susceptibility of human cells may be regulated by genes in the HLA region on chromosome 6 . Proc Natl Acad Sci U S A. 1986;83(15):5688-92.

15. Moretta A, Tambussi G, Bottino C, Tripodi G, Merli A, Ciccone E, Pantaleo G, Moretta L. A novel surface antigen expressed by a subset of human CD3- CD16+ natural killer cells. Role in cell activation and regulation of cytolytic function. $J$ Exp Med. 1990;171(3):695-714.

16. Liu WR, Kim J, Nwankwo C, Ashworth LK, Arm JP. Genomic organization of the human leukocyte immunoglobulin-like receptors within the leukocyte receptor complex on chromosome 19q13.4. Immunogenetics. 2000;51(8-9):659-69.

17. Gonzalez A, McErlean C, Meenagh A, Shovlin T, Middleton D. Killer cell immunoglobulin-like receptor allele discrimination by high-resolution melting. Hum Immunol. 2009;70(10):858-63.

18. Uhrberg $M$, Valiante NM, Shum BP, Shilling HG, LienertWeidenbach K, Corliss B, Tyan D, Lanier LL, Parham P. Human diversity in killer cell inhibitory receptor genes. Immunity. 1997;7(6):753-63.

19. Middleton D, Gonzelez F. The extensive polymorphism of KIR genes. Immunology. 2010;129(1):8-19.

20. Towner P. Purification of DNA. Essential Molecular Biology. Ed by Brown TA. Oxford, Oxford University Press, 1995: 47-54.

21. Spiroski M, Arsov T, Petlichkovski A, Strezova A, Trajkov D, Efinska-Mladenovska O, Zaharieva E. Case Study: Macedonian Human DNA Bank (hDNAMKD) as a source for public health Genetics. Health Determinants in the Scope of
New Public Health. B. G. Georgieva L. Sofia, Hans Jacobs, 2005.

22. Marsh SG, Parham P, Dupont B, Geraghty DE, Trowsdale J Middleton D, Vilches C, Carrington M, Witt C, Guethlein LA, Shilling $H$, Garcia CA, Hsu KC, Wain H. Killer-cell immunoglobulin-like receptor (KIR) nomenclature report, 2002. Immunogenetics. 2003;55(4):220-6.

23. Robinson J, Mistry K, McWilliam H, Lopez R, Marsh SG. IPD-the Immuno Polymorphism Database. Nucleic Acids Res. 2010;38(Database issue):D863-9.

24. Gómez-Lozano N, Vilches C. Genotyping of human killer-cel immunoglobulin-like receptor genes by polymerase chain reaction with sequence-specific primers: an update. Tissue Antigens. 2002;59(3):184-93.

25. Excoffier L, Laval G, Schneider S. Arlequin (version 3.0): an integrated software package for population genetics data analysis. Evol Bioinform Online. 2007;1:47-50.

26. Mattuiz PL, Inde D, Piazza A, Ceppellini R, Bodmer WF. New approaches to population genetics and segregation analysis of the HLA system. In: TerasakiPI, editor. Histocompatibility testing. Copenhagen: Munksgaard; 1970. p. 193-205.

27. Lewontin RC, Dunn LC. The Evolutionary Dynamics of a Polymorphism in the House Mouse. Genetics. 1960;45(6):70522.

28. Lewontin RC. The Interaction of Selection and Linkage. I. General Considerations; Heterotic Models. Genetics. 1964;49(1):49-67.

29. Djulejic E, Petlichkovski A, Trajkov D, Hristomanova S, Middleton D, Spiroski M. Distribution of killer cell immunoglobulinlike receptors in the Macedonian population. Hum Immunol. 2010;71(3):281-8.

30. Gonzalez-Galarza FF, Christmas S, Middleton D, Jones AR Allele frequency net: a database and online repository for immune gene frequencies in worldwide populations. Nucleic Acids Res. 2011;39(Database issue):D913-9.

31. Gourraud PA, Gagne K, Bignon JD, Cambon-Thomsen A, Middleton D. Preliminary analysis of a KIR haplotype estimation algorithm: a simulation study. Tissue Antigens. 2007;69 Suppl 1:96-100.

32. Denis L, Sivula J, Gourraud PA, Kerdudou N, Chout R, Ricard C, Moisan JP, Gagne K, Partanen J, Bignon JD. Genetic diversity of KIR natural killer cell markers in populations from France, Guadeloupe, Finland, Senegal and Réunion. Tissue Antigens. 2005;66(4):267-76

33. Bontadini A, Testi M, Cuccia MC, Martinetti M, Carcassi C, Chiesa A, Cosentini E, Dametto E, Frison S, lannone AM, Lombardo C, Malagoli A, Mariani M, Mariotti L, Mascaretti L, Mele L, Miotti V, Nesci S, Ozzella G, Piancatelli D, Romeo G, Tagliaferri C, Vatta S, Andreani M, Conte R. Distribution of killer cell immunoglobulin-like receptors genes in the Italian Caucasian population. J Transl Med. 2006;4:44

34. Niokou D, Spyropoulou-Vlachou M, Darlamitsou A Stavropoulos-Giokas C. Distribution of killer cell immunoglobulin-like receptors in the Greek population. Hum Immunol. 2003;64(12):1167-76.

35. Verheyden S, Bernier M, Demanet C. Identification of natural killer cell receptor phenotypes associated with leukemia Leukemia. 2004;18(12):2002-7.

36. Cook MA, Moss PA, Briggs DC. The distribution of 13 killer-cell immunoglobulin-like receptor loci in UK blood donors from three ethnic groups. Eur J Immunogenet. 2003;30(3):213-21.

37. Suzuki Y, Hamamoto Y, Ogasawara Y, Ishikawa K, Yoshikawa Y, Sasazuki T, Muto M. Genetic polymorphisms of killer cell immunoglobulin-like receptors are associated with susceptibility to psoriasis vulgaris. J Invest Dermatol. 2004;122(5):1133-6. 
38. Petlichkovski A, Djilejic E, Trajkov D, Efinska-Mladenovska O, Hristomanova S, Kirijas M, Senev A, Spiroski M. Distribution of killer cell immunoglobulin-like receptor genes in Roma from Republic of Macedonia. Int J Immunogenet. 2011; 38 (6) 493500

39. Norman PJ, Carrington CV, Byng M, Maxwell LD, Curran MD, Stephens HA, Chandanayingyong D, Verity DH, Hameed K, Ramdath DD, Vaughan RW. Natural killer cell immunoglobulinlike receptor (KIR) locus profiles in African and South Asian populations. Genes Immun. 2002;3(2):86-95.

40. Rajalingam R, Krausa $P$, Shilling $H G$, Stein JB, Balamurugan A, McGinnis MD, Cheng NW, Mehra NK, Parham P. Distinctive KIR and HLA diversity in a panel of north Indian Hindus. Immunogenetics. 2002;53(12):1009-19.

41. Spiroski M, Milenkovic Z, Petlichkovski A, Ivanovski L, Topuzovska IK, Djulejic E. Killer cell immunoglobulin-like receptor genes in four human West Nile virus infections reported 2011 in the Republic of Macedonia. Human immunology. 2013; 74(3), 389-394.

42. Petlichkovski A, Stojanoski Z, Djulejic E, Georgievski B, Spiroski M. Association of Killer Cell Immunoglobulin-Like Receptor Genes with the Graft versus Host Disease after Related Haematopoietic Stem Cell Transplantation in Patients with Haematological Malignancies from Republic of Macedonia. Maced J Med Sci. 2012; 5(4):404-410.

43. Petlichkovski A, Milenkovic Z, Djulejic E, Jefremovska B, Babacic H, Ivanovski LJ, Kondova Topuzovska I, Spasovska K, Spiroski M. Association of Killer Cell Immunoglobulin-Like Receptor Genes with Pandemic Influenza A (H1N1)pdm09 Infection in Critically III Macedonian Patients. Maced J Med Sci. 2012; 5(3):288-295

44. Petlichkovski A, Stojanoski Z, Cibrev D, Spiroski M. Killer Cell Immunoglobulin-Like Receptor Genes Polymorphisms in Macedonian Patients with Haematological Malignancies. Maced J Med Sci. 2013; 6(1):24-30.

45. Djulejic E, Petlichkovski A, Trajkov D, Dimitrov G, Alabakovska $\mathrm{S}$. KIR Gene Frequencies in Women with Infertility Problems. SEE J Immunol. 2015; 2015:20002.

46. Single RM, Martin MP, Gao X, Meyer D, Yeager M, Kidd JR Kidd KK, Carrington M. Global diversity and evidence for coevolution of KIR and HLA. Nat Genet. 2007;39(9):1114-9.

47. Middleton DAM, Serrano-Vela JI, Moscosoc J, Antonio ArnaizVillena A. Different Evolution of Inhibitory and Activating Killer Immunoglobulin Receptors (KIR) in Worldwide Human Populations. Open Immunol J. 2008;1:42-50. 